\title{
Erratum to: 8th annual meeting of Chinese College of Surgeons and 19th annual meeting of the European Society of Surgery
}

\author{
Akif Enes ARIKAN
}

Published online: 25 July 2015

(C) Springer-Verlag Wien 2015

\section{Erratum to: Eur Surg (2015) 47 (Suppl 1):S1-S281 DOI 10.1007/s10353-015-0305-0}

For abstract "Incidental gallbladder cancer: 16 years Cerrahpasa experience" and "New imaging technique for breast carcinoma; Cerrahpasa experience. Preliminary Report" the authors and affiliations are missing.

Incidental gallbladder cancer: 16 years Cerrahpasa experience

Suleyman DEMIRYAS MD', Ozan AKINCI MD ${ }^{1}$, Serkan TEKSOZ Assoc. Prof. MD, FEBES', Yasemin KUCUK MD ${ }^{1}$, Akif Enes ARIKAN MD ${ }^{1, *}$, Nuray KEPIL MD ${ }^{2}$, Ihsan TASCI Prof. MD ${ }^{1}$

${ }^{1}$ Department of General Surgery, Istanbul University, Cerrahpasa Medical Faculty, Istanbul, Turkey *e-mail: enesarikan@yahoo.com

2Department of Pathology, Istanbul University, Cerrahpasa Medical Faculty, Istanbul, Turkey
New imaging technique for breast carcinoma; Cerrahpasa experience. Preliminary Report

\footnotetext{
Murat OZCAN Prof. MD', Mehmet CAYOREN PhD', Akif Enes ARIKAN MD ${ }^{1, *}$, Mahmut Alp KILIC MSc ${ }^{3}$, Serkan TEKSOZ Assoc. Prof. MD, FEBES ${ }^{1}$, Sina FERAHMAN MD', Ibrahim AKDUMAN Prof. PhD ${ }^{2}$, Tunaya KALKAN Prof.PhD

${ }^{1}$ Department of General Surgery, Istanbul University, Cerrahpasa Medical Faculty, Istanbul, Turkey *e-mail: enesarikan@yahoo.com

${ }^{2}$ Department of Electronics and Communication Engineering, Istanbul Technical University, Engineering Faculty, Istanbul, Turkey ${ }^{3}$ Department of Biophysics, Istanbul University, Cerrahpasa Medical Faculty, Istanbul, Turkey
}

The online version of the original article can be found under doi:10.1007/s10353-015-0305-0

A. E. ARIKAN, M.D. $(\bowtie)$

Department of General Surgery,

Istanbul University, Cerrahpasa Medical Faculty,

Istanbul, Turkey

e-mail: enesarikan@yahoo.com 